\title{
L'inquisition face aux Lumières et à la révolution française en Nouvelle-Espagne : le dossier et le procès d'Esteban Morel (1781-1795)
}

The Inquisition, the Enlightenment, and the French Revolution in New Spain : the dossier and the trial of Esteban Morel (1781-1795)

Liliana Schifter, Patricia Aceves et Patrice Bret

\section{(2) OpenEdition}

Journals

\section{Édition électronique}

URL : https://journals.openedition.org/ahrf/12117

DOI : $10.4000 /$ ahrf. 12117

ISSN : 1952-403X

Éditeur :

Armand Colin, Société des études robespierristes

Édition imprimée

Date de publication : 1 septembre 2011

Pagination : 103-127

ISBN : 978-2-200-92700-4

ISSN : 0003-4436

\section{Référence électronique}

Liliana Schifter, Patricia Aceves et Patrice Bret, «L'inquisition face aux Lumières et à la révolution française en Nouvelle-Espagne : le dossier et le procès d'Esteban Morel (1781-1795) », Annales historiques de la Révolution française [En ligne], 365 | Juillet-septembre 2011, mis en ligne le 01 septembre 2014, consulté le 23 avril 2022. URL : http://journals.openedition.org/ahrf/12117 ; DOI : https://doi.org/10.4000/ahrf.12117 


\title{
L'INQUISITION FACE AUX LUMIÈRES ET Ȧ LA RÉVOLUTION FRANÇAISE EN NOUVELLE-ESPAGNE : LE DOSSIER ET LE PROCÈS D'ESTEBAN MOREL (1781-1795)
}

\author{
Liliana SCHIFTER \\ Patricia ACEVES \\ Patrice BRET
}

\begin{abstract}
Dans les décennies qui précédèrent l'indépendance du Mexique les sciences et les techniques furent un vecteur privilégié des Lumières mais, parallèlement aux réformes institutionnelles éclairées en la matière, l'Inquisition exerçait une étroite surveillance sur les milieux intellectuels. Venu de Guadeloupe après être passé dans plusieurs colonies espagnoles, Esteban Morel, un médecin français installé en Nouvelle-Espagne, en fut victime lors de la répression contre les résidents français après la guerre entre la République et l'Espagne. Introducteur de l'inoculation dans la vice-royauté (1779) et propriétaire de mines innovant, il appartenait aux cercles éclairés de la capitale coloniale, contribuant aux débats dans la presse locale et à l'exploitation minière, première richesse du pays. L'influence de ce lecteur de Voltaire et de Buffon, déiste et probablement francmaçon, était d'autant plus dangereuse qu'elle s'exerçait jusque dans l'entourage du Vice-Roi. Au-delà du cas particulier, le dossier que l'Inquisition forma contre Morel dès 1781 illustre les formes discrètes de la pénétration des Lumières au Mexique, les résistances auxquelles elles furent confrontées et la violence de la répression en matière religieuse, qui s'accentua avec la crainte d'une conspiration révolutionnaire en 1794 .
\end{abstract}

Mots-clés : Esteban Morel, Inquisition, inoculation, NouvelleEspagne, Mexique. 
La question de l'impact de la Révolution française sur l'indépendance du Mexique a fait l'objet de nombreuses études des deux côtés de 1'Atlantique. L'ouvrage classique très documenté de Nicolás Rangel en 1929 faisait même de la Révolution française la cause première des émancipations américaines ${ }^{1}$. Cette perspective largement partagée par l'historiographie conservatrice ou libérale a été nuancée depuis, avec le Bicentenaire de la Révolution ${ }^{2}$, qui a fait émerger de nouvelles sources ${ }^{3}$, notamment par l'étude de la présence française en Nouvelle-Espagne ${ }^{4}$, de l'Inquisition ${ }^{5}$ et des affaires judiciaires ${ }^{6}$. Elle a été récemment critiquée par Gabriel Torres Puga qui a étudié de près les poursuites contre les Français du Mexique en 1794-17957. En reprenant ici l'examen du cas de l'une des victimes de ces persécutions, notre objectif est de cerner des modes possibles de pénétration de la pensée des Lumières et des idéaux de la Révolution fran-

(1) Nicolás RANGEL, Los precursores ideológicos de la guerra de Independencia 17891794, México, Publicaciones del Archivo General de la Nación, 1929-1932, 2 vol. : I - La revolución francesa, una de las causas externas del movimiento insurgente; II - La masoneria en Mexico. Siglo XVIII. Voir aussi Raúl CARDIEL ReYEs, La primera conspiración por la Independencia de México, Mexico, Secretaría de Educación Pública, 1982.

(2) François-Xavier Guerra (dir.), L'Amérique latine face à la révolution française (actes du colloque de l'Afssal), t. I, Caravelle. Cahiers du monde hispanique et luso-brésilien, $\mathrm{n}^{\circ}$ 54, 1990; Solange Alberro et al. (dir.), La Revolución Francesa en México, México, El Colegio de México/ Centro de Estudios Mexicanos y Centroamericanos, 1993.

(3) Francisco Javier CASADO, «Fondos americanistas de la sección de Estado del Archivo Histórico Nacional de Madrid: la presencia francesa en Nueva España en el último cuarto del siglo XVIII ", Estudios de historia social y económica de América. Revista de la Universidad de Alcalá, $\mathrm{n}^{\circ} 11,1989$, p. 365-374.

(4) Jacques Houdaille, «The Frenchmen and Francophiles in New Spain from 1760 to 1780 », The America's Quaterly Review of Inter-American Culturel History, 13, 1956, p. 1-29; Frédérique LANGUE, «Les Francais en Nouvelle Espagne à la fin du XVIII siècle : médiateurs de la Révolution ou "nouveaux créoles"? », dans François-Xavier GuERRA (dir), L'Amérique latine face à la révolution, op. cit., p. 37-60.

(5) Georges Baudot, María Águeda Méndez, « La Revolución francesa y la Inquisición mexicana. Textos y pretextos », dans François-Xavier GuERra (dir), L'Amérique latine face à la révolution, op. cit., p. 89-105; Gabriel Torres PugA, Los últimos años de la Inquisición en Nueva España, Mexico, Miguel Angel Porrúa - INAH, 2004.

(6) Antonio IBARRA, «Conspiración, desobediencia social y marginalidad en la Nueva España : la aventura de Juan de la Vara », Historia Mexicana, XLVII, 1, 185, 1997, p. 5-34; id., « La persecución institucional de la disidencia novohispana : patrones de inculpación y temores políticos de una época ", dans Felipe CASTRo et Marcela Terrazas (dir.), Disidencia y disidentes en la historia de México, Mexico, UNAM-IIH, 2004.

(7) Gabriel Torres PugA, « Centinela mexicano contra francmasones : un enredo detectivesco del Licenciado Borunda en las causas judiciales contra franceses de 1794 », Estudios de Historia Novohispana, México, UNAM-IIH, ENH, n 33, juillet-décembre 2005, p. 57-94. - Depuis la rédaction de notre article, l'auteur a intégré ces données dans une perspective plus large : Gabriel Torres Puga, Opinión pública y censura en Nueva España. Indicios de un silencio imposible (17671794), Mexico, El Colegio de México, 2010. 
çaise, ainsi que leurs limites. En l'occurrence, le médecin qui vécut une trentaine d'années dans l'Amérique espagnole, dont plus de seize en Nouvelle Espagne, sous le nom hispanisé d'Esteban Morel, fut l'un de ceux qui eurent le plus d'influence, par ses liens étroits avec les élites locales ${ }^{8}$.

Depuis cet exil choisi, Morel vécut la révolution américaine et la Guerre d'Indépendance des États-Unis, puis la fin de la monarchie et l'instauration de la République en France, qui provoquèrent l'inquiétude des grandes puissances en Europe et de leurs possessions d'Amérique. L'infiltration des idées révolutionnaires dans les colonies était une préoccupation constante pour les Bourbons d'Espagne. La diffusion de l'information passait par des intermédiaires, majoritairement français, qui l'apportaient ou l'importaient en Amérique, où leurs compatriotes la faisaient circuler. Toutes les nouvelles provenant de France n'étaient certes pas politiques. Les unes avaient un caractère familial, culturel ou commercial, d'autres concernaient les nouveautés scientifiques ou techniques, mais elles véhiculaient aussi des courants de pensée et des idées bien souvent en contradiction avec les préceptes de l'Église catholique, qui se chargea de les combattre sans trêve'.

C'est dans ce contexte que Morel acquit réputation, position sociale et richesse au Mexique.

\section{De Montpellier à Mexico : itinéraire d'un médecin des Lumières}

Estienne Henri Maurel naquit à Aubagne le 14 janvier 1741 dans une famille probablement liée au négoce, assez aisée pour lui offrir des études d'un coût élevé ${ }^{10}$. Selon ses propres déclarations, après avoir fait sa philosophie et sa grammaire à Marseille ${ }^{11}$, il aurait poursuivi des

(8) Liliana Schifter, Medicina, minería e Inquisición en La Nueva España : Esteban Morel (1744-1795), México, UAM-X, SQM, CNQFB, 2002 (Biblioteca de Historia de la Farmacia 3). Sur le contexte savant, voir Patricia ACEves, Química, Botánica y Farmacia en la Nueva España a finales del Siglo XVIII, México, UAM-X, 1993.

(9) Solange Alberro et al., La Revolución Francesa en México; Frédérique Langue, « Los franceses en la Nueva España... ».

(10) Et ni en 1730 à Marseille (Diccionario Porrúa de historia, biografia y geografía de México, 6éd., Mexico, Editorial Porrúa, 1995, t. 3, p. 2356-2357), ni en 1744 à « Aubarre » (déclaration à l'Inquisition en 1794 avec mauvaise lecture de la graphie « Aubañe »). Il est fils de Cosme Maurel et Marie Beaumond (Arch. Dép. Bouches-du-Rhône).

(11) Archivo General de la Nación, Mexico [AGN], Inquisición, année 1795, vol. 1379, exp. 11 : « Relación de la causa de fe q[u]e en este Trib[una]l ha seguido el S[eño]r Fiscal con el D[octo]r D[o]n Esteban Morel de Nacion frances medico de profesion, viudo de Da Agueda Romero Española y v[ecin]a que fue de la Ciudad de Banquisimeto en la Prov[inci]a de Venezuela, por proposiciones », f. $228-286$ (f. $269 v$ ). 
études médicales à Aix-en-Provence, puis à Montpellier ${ }^{12}$. Cette dernière faculté renommée était alors troublée par des luttes intestines, mêlant des rivalités personnelles ou générationnelles aux querelles scientifiques. En chimie, la chaire était occupée depuis 1732 par Fizes qui dispensait en latin un enseignement fidèle aux théories médicales de Boerhaave et aux idées mécanistes de Chirac, et il fallut offrir une chaire de médecine non spécialisée au chimiste Venel en 1759 pour introduire un enseignement en français et la théorie de Stahl $1^{13}$. Venel était le principal rédacteur des articles de chimie de l'Encyclopédie de Diderot et D'Alembert, dont d'autres collaborateurs appartenaient au corps professoral montpelliérain, tels le médecin Barthez, fondateur du vitalisme, et le pharmacien Montet. Selon une pratique courante, Maurel suivit aussi des cours particuliers en marge de l'université, notamment ceux d'anatomie chez Davizard et chez Vigaroux, de pharmacie chez Lapeyre, apothicaire de la Ville, et ceux de chimie de Venel dans le laboratoire de Montet ${ }^{14}$.

Bien que ses titres aient été vérifiés et validés plus tard par les autorités médicales mexicaines, une incertitude plane sur la formation de Maure ${ }^{15}$. Effectivement inscrit d'août 1760 à janvier 1762, il ne suivit que six des douze trimestres requis et n'apparaît pas sur les registres d'examens ${ }^{16}$. Il ne fut donc ni bachelier, ni a fortiori licencié, titre qui ouvrait le droit d'exercer la médecine, ni docteur de Montpellier. Certes, par suite du coût élevé des études régulières, des subsistances et des cours privés, ce type de cursus écourté n'était pas rare ${ }^{17}$.

(12) Ibid.; AGN, Hospitales, année 1783, vol. 47, exp. 29 : Morel, « Solicitud de Esteban Morel a la plaza de médico al Hospital Real de Naturales », f. 464-473 (f. 465).

(13) L'ouvrage de référence est Louis DuLIEu, La Médecine à Montpellier, Tome III, L'Époque Classique, $2^{e}$ partie, Avignon, Les Presses Universelles, 1973. Sur Venel et sur le contexte de la chimie à Montpellier, voir Christine LeCornu-Lehman, « Gabriel-François Venel (1723-1775). Sa place dans la chimie française du XVIII ${ }^{\mathrm{e}}$ siècle "), thèse de l'Université Paris X-Nanterre, 2006, surtout p. 76-102.

(14) Esteban Morel, « Solicitud... », f. 465v. Christine Lehman a édité le cours de Venel de 1761, qu'a pu suivre Maurel (Venel, Cours de chimie, [Dijon], Corpus / EUD, 2010). Sur les autres professeurs de Morel, voir Liliana SCHIFTER, Medicina, minería e Inquisición, op. cit., p. 33-36.

(15) Le 17 mars 1783, il écrit avoir été reçu docteur à Montpellier dix-neuf ans plus tôt (Morel, « Solicitud... », f. 465, 466), mais semblerait bachelier et docteur d'Aix par sa déposition du 3 octobre 1794, titres non décernés en médecine par cette université («Relacion de la causa... », f. 269v).

(16) Il signe les registres d'inscriptions trimestrielles (sixième en novembre 1761), mais ne figure pas sur ceux d'examens (Bibliothèque interuniversitaire, Section médecine, Archives de la faculté de médecine, S 31). Nous remercions Jacqueline Mathieu et Mireille Vial d'avoir vérifié ce point.

(17) En trois ans, les études d'un bachelier coûtaient quelque 600 livres (150 livres de droits d'entrée et frais d'inscription aux examens, autant pour les «pourboires et autres reconnaissances » et 300 pour les besoins de l'étudiant). Les cours particuliers venaient en sus : celui de Venel coûtait deux louis, soit 96 livres (Christine Lehman, Venel, op. cit., p. 236). 
$\mathrm{Au}$ demeurant, ces titres n'étant pas nécessaires pour obtenir une place dans la médecine militaire, Maurel fut envoyé en 1765 en Guadeloupe avec une commission de médecin du Roi pour exercer le service dans les hôpitaux militaires ${ }^{18}$. Faut-il voir dans ce départ pour les Antilles l'influence du professeur de Lamure, natif de la Martinique ${ }^{19}$, dont il avait suivi non seulement le cours de physiologie à l'université, mais aussi le cours particulier d'institutions médicales? Ou avait-il déjà des liens familiaux dans l'île? Quoi qu'il en soit, outre la Guadeloupe, il servit également en Martinique et à la Marguerite. Se lassa-t-il du poids de la tâche de médecin aux colonies pour un jeune sans expérience ${ }^{20}$ ? Eut -il des démêlés avec les autorités? Ou saisit-il une occasion de carrière aventureuse aux perspectives plus prometteuses? Toujours est-il que, pour des raisons indéterminées, Maurel passa très vite dans l'empire espagnol ${ }^{21}$.

À La Guaira, en Nouvelle-Grenade, il épousa une créole du Venezuela qui le dénonça au commissariat de Caracas en mars 1771 pour sa « conduite irrévérencieuse » en matière de religion ${ }^{22}$. Puis, après quelques années à La Havane, il gagna la Louisiane - cédée à la couronne espagnole en 1763 - où le gouverneur l'autorisa en juin 1777 à exercer à l'hôpital de la Charité de la Nouvelle-Orléans. Mais il n'y resta guère et se fixa l'année suivante au Mexique, qu'il ne devait plus quitter.

La puissante colonie de Nouvelle-Espagne offrait sans doute davantage d'attraits. Parvenue au premier rang mondial pour la production d'argent, elle était alors le théâtre d'un essor sans précédent. Hors de la sphère économique, le développement du pays se manifestait aussi dans le secteur culturel, où un groupe de savants et hommes de lettres éclairés s'efforçait d'introduire les derniers progrès dans leurs domaines respectifs $^{23}$. Malgré sa situation périphérique, le Mexique était intégré au réseau

(18) Il quitta la France en 1765 (« Relacion de la causa... », fo 269v). Il n’y a aucune trace de Maurel dans les archives administratives des colonies (communication de François Regourd à P. Bret, 8 mai 2001).

(19) Louis DuliEu, « François-Bourguignon de Bussières de Lamure (1717-1787) », Revue d'histoire des sciences, 21-3 (1968), 233-244.

(20) Outre ses consultations - jusqu'à 300 par jour, dit-il - il devait pratiquer des opérations chirurgicales, faire des inspections de médicaments dans les pharmacies et examiner les aspirantsmédecins.

(21) Depuis « environ 29 ans » en 1794 (« Relacion de la causa... », f. 268v).

(22) AGN, Inquisición, année 1795, vol. 1321, exp. 2, Inquisición de Cartagena : « Sobre los asuntos seguidos en la causa de Don Esteban Morel », f. 4-5.

(23) Patricia Aceves, Química, Botánica y Farmacia en la Nueva España a finales del Siglo XVIII, México, UAM-X, 1993. 
international d'échanges scientifiques et il revint à Morel d'être témoin et acteur d'importants projets et de participer à la mise en place d'institutions savantes modernes.

Morel arrivait avec une bonne recommandation puisque l'Inspecteur Général José de Gálvez - l'artisan des réformes bourboniennes en Amérique - autorisa en personne son entrée sur le territoire néohispanique. Jouissant de la faveur et de la confiance des autorités, il n'eut aucun problème pour faire valider ses titres par les plus hautes instances médicales (Real Tribunal del Protomedicato) et pour exercer sa profession librement ${ }^{24}$. Il séjourna brièvement à Guanajuato, devenu le principal centre minier argentifère depuis le déclin des mines de Potosi, et à Real de Catorce. Là, tout en menant son activité de médecin, il noua des premiers contacts avec le secteur minier. Il les renforça plus tard en devenant fondé de pouvoirs de Don Anselmo Montero, maître de la mine du Morán à Real Del Monte, près de Pachuca, l'une des plus riches de l'époque (1789). Quand il en fut devenu lui-même propriétaire, un rival contesta devant le Tribunal royal des mines la possibilité pour un étranger de posséder une mine (1793). Morel obtint gain de cause, puisqu'un décret royal du 27 novembre 1791 lui avait octroyé le statut d'" étranger toléré » au Mexique et donc autorisé à acquérir une mine et le mécanicien et mathématicien Diego de Guadalajara Tello lui fit les plans d'une machine pour l'assèchement de la mine ${ }^{25}$.

De nature entreprenante, Morel déploya une grande activité dans tous ses centres d'intérêt - les aspects théoriques et pratiques de la médecine, de la pharmacie et de l'exploitation des mines - et sut s'intégrer rapidement aux élites éclairées des secteurs administratif et savant de la colonie, dans lesquels il se constitua de puissantes amitiés et diffusa de manière privilégiée la science et la culture française. En 1788, il pouvait, sans modestie, s'enorgueillir publiquement d'appartenir à un cercle de savants illustres par leurs bonnes manières et leur science :

«Personne parmi tous ceux qui en ont connaissance ne doutera de la vénération avec laquelle j'ai toujours considéré les personnes de cette Cour; savants auxquels je me frotte le plus, essayant de m'améliorer par

(24) Esteban Morel, « Solicitud... », f. 464-473.

(25) Voir plus loin la suite de ce litige (Archivo Histórico del Palacio de Minería, Mexico, Morán, De, mina, Real del Monte, Hidalgo [1794-II 69d.22]; Liliana SCHIFTER, Medicina, minería e Inquisición..., p. 41-45). Cette mine appartint plus tard à Fausto d'Elhuyar associé au Britannique Thomas Murphy. 
leur fréquentation. Je nommerais beaucoup d'entre eux, avec l'éloge qui leur est dû, si ne m'arrêtait la crainte d'offenser leur modestie $»^{26}$.

Morel fut particulièrement lié aux deux principaux savants de la colonie, le prêtre créole José Antonio de Alzate y Ramírez, seul correspondant de l'Académie des sciences de Paris dans l'Amérique espagnole ${ }^{27}$, et l'astronome et physicien Joaquin Velázquez de León, compagnon de l'abbé Chappe d'Auteroche pour l'observation, en Basse-Californie, du passage de Vénus devant le soleil en 1769. Avec eux, il débattit publiquement des techniques minières dans les journaux locaux comme la Gaceta de Literatura, éditée par le premier, et la Gaceta de México ${ }^{28}$. Chez eux ou dans des réunions, il rencontra aussi quatre savants natifs d'Espagne, que la Couronne chargea de mettre en œuvre les projets de réforme bourboniens et qui contribuèrent à la diffusion des dernières avancées scientifiques de l'époque : le minéralogiste Fausto de Elhúyar, directeur général des mines de Nouvelle-Espagne, venu à la tête d'une mission d'ingénieurs saxons pour introduire la méthode d'amalgamation du Baron Ignaz von Born $^{29}$; le mathématicien et architecte Miguel Constanzó, titulaire de la chaire de mathématiques à l'Académie Saint-Charles (Real Academia de San Carlos) et directeur des Pavés et Travaux publics (Empedrados y Obras Públicas); le pharmacien Vicente Cervantes, titulaire de la chaire de botanique du Jardin botanique royal de Mexico (Real Jardín Botánico),

(26) Esteban Morel, « Respuesta del Dr. Esteban Morel a la carta de D. José, Alzate que salió en suplemento de la Gaceta de 29 de diciembre último pasado, y a algunos párrafos de un papel anónimo del mismo dia », dans Gacetas de Literatura, op. cit., p. 365.

(27) Patrice Bret, «Alzate y Ramírez et l'Académie royale des sciences de Paris : la réception des travaux d'un savant du Nouveau monde », dans Patricia Aceves Pastrana (dir.), Periodismo cientifico en el siglo XVIII : José Antonio de Alzate y Ramírez (Actes de la VIII ${ }^{\mathrm{e}}$ Réunion de la RIHECQB, Mexico, 24-26 novembre 1999), Mexico, Universidad autónoma metropolitana-Xochimilco, 2001, p. 123-205; id., « Interplay between European and Colonial Journals : José Antonio de Alzate's learned periodicals in New Spain, 1768-1795 », dans Andrea STEINER (éd.), Interplay between Journals, à paraître.

(28) Esteban Morel, « Historia natural y carta del Dr. Esteban Morel al autor sobre el malacate », dans Gacetas de Literatura, Puebla, Reimpresas en la Oficina del Hospital de San Pedro, 1831, t. 4, p. 300; « Respuesta de D. Esteban Morel al autor », ibid., p. 358.

(29) Francisco ARAGón de LA CRUZ, « La expedición de los mineros sajones a Hispanoamérica (1788-1810), una confrontación tecnológica en los métodos de amalgación de los minerales de plata », dans Mari Álvarez Lires et al., Estudios de Historia das Ciencias e das Técnicas, Pontevedra, 2001, Servicio de publicaciónes, Deputación provincial de Pontevedra, t. I., p. 283-294 
introducteur de la nomenclature de Linné ${ }^{30}$; et le métallurgiste Francisco Xavier Sarría, directeur de la Loterie Royale de la Nouvelle Espagne, mais également métallurgiste de solide formation mathématique et chimique, qui introduisit en 1791 les théories de Lavoisier au Mexique en donnant un supplément à son traité de métallurgie de $1784^{31}$. Elhúyar et Sarría étaient également propriétaires de mines, comme Morel. Mais celui-ci était avant tout médecin. L'un des épisodes marquants de sa carrière fut l'introduction de l'inoculation de la variole au Mexique.

\section{Introduction de l'inoculation de la variole au Mexique}

L'inoculation était l'une des grandes questions de santé publique en Europe. Saluée entre autres par Buffon ou Diderot, elle s'imposa en France lorsque, le 18 juin 1774, Lieutaud fit inoculer les enfants royaux vingt ans après le "Mémoire sur l'inoculation de la variole» de La Condamine à l'Académie des sciences, mais l'Espagne était restée très en retrait de ce mouvement ${ }^{32}$. Pour enrayer la terrible épidémie qui dévasta la Nouvelle Espagne en 1779, la mairie de Mexico chargea Morel de rédiger un rapport sur l'utilité de cette méthode et de conduire les expérimentations nécessaires pour faire toute la lumière sur son emploi ${ }^{33}$. C'est ainsi qu'il réalisa la première inoculation de la variole à Mexico.

La «bienheureuse» (afortunada) fut Doña Barbara Rodriguez de Velasco, inoculée le 4 octobre 1779. Treize autres sujets suivirent, dont une fillette à la demande de José Antonio de Alzate y Ramírez, les deux

(30) Voir Patricia Aceves, Química, Botánica y Farmacia, op. cit.; id., « Botánica, Farmacia y Química en México : Vicente Cervantes (1787-1829)», dans María Teresa Miras Portugal, Antonio GonzÁlez Bueno et Antonio DoAdrio Villarejo (éds.), El 250 Aniversario del nacimiento de Vicente Cervantes (1758-1829) : relaciones cientificas y culturales entre España y América durante la Ilustración, Madrid, Real Academia Nacional de Farmacia, 2009, p. 101-116.

(31) Francisco Xavier SARrí, Suplemento al Ensayo de Metalurgia, Mexico, Felipe de Zúñiga y Ontiveros, 1791. Voir Patricia Aceves, «L'enseignement et l'application de la nouvelle chimie au Mexique au temps de Lavoisier », dans Patrice BRET (dir.), « Débats et chantiers autour de Lavoisier et de la révolution chimique », Revue d'Histoire des Sciences, XLVIII/1-2 (1995), 123-132; id., « Fuentes para la historia de la química : la obra de Francisco Xavier Sarría », dans Centenario Simão Mathias : Documentos, Métodos e Identidade da Historia da Ciencia, Sao Paulo, PUC-SP, 2009, p. 243-244.

(32) P.-J. GuINARD, « Notes sur l'inoculation de la variole en Espagne », dans Mélanges à la mémoire de Jean Sarrailh, Paris, Centre de recherches de l'Institut d'études hispaniques, t. I, 1966; Juan Tomado de Riera et Juan Granda-Juesas, La inoculación de la viruela en la España Ilustrada, Valladolid, Universidad de Valladolid, 1987.

(33) Francisco FernÁndez del Castillo, Los viajes de Don Francisco de Xavier de Balmis. Notas para la historia de la expedición vacunal de España a América y Filipinas (1803-1806), $2^{\mathrm{e}}$ éd. Mexico, Sociedad Médica Hispano Mexicana, 1985, p. 23; Liliana SchIFTER, Medicina, minería e Inquisición, op. cit., p. 17. 
enfants de Pierre Lafargue, un commerçant français de la ville, et six petits indiens qu'il traita chez lui afin de rassurer la population indigène en lui donnant des exemples de la réussite de l'opération. Tous survécurent au traitement sans complications majeures ${ }^{34}$. Ces succès incitèrent le Vice-Roi et le maire à ouvrir, sous la responsabilité de Morel, un « hôpital d'inoculation » au sein du nouvel hôpital installé au couvent de San Hipolito deux ans plus tôt. Premier acte d'un appui des autorités en la matière, l' « Avis au public » annonçant l'inauguration de cette clinique le $1^{\text {er }}$ novembre 1779 mettait en avant les « admirables effets qu'a produits dans beaucoup de pays d'Asie, d'Afrique, d'Europe, dans certains d'Amérique, et encore dans cette Ville, l'inoculation de la Variole, non seulement adoptée, mais exécutée sur les Souveraines Personnes des Rois $\rangle^{35}$.

Le rapport de 125 pages que Morel présenta devant les membres de la mairie en 1780, sous le titre «Dissertation sur l'utilité de l'inoculation », est l'œuvre d'un homme éclairé, érudit et moderne, très au fait de la littérature européenne sur le sujet, la plus libre (article « Inoculation » de l'Encyclopédie) et la plus récente (cahier de janvier $1779 \mathrm{du}$ Journal de médecine de Paris $)^{36}$. Introduisant ainsi en Nouvelle Espagne le travail de La Condamine, qui n'avait pas été diffusé en Espagne, il démontre scientifiquement les avantages de l'inoculation pour protéger la population de l'épidémie : «Le très sage auteur de tout a donné à l'homme l'entendement pour sa conservation [...] et la raison clame en faveur de l'inoculation $\gg{ }^{37}$. Morel fait une analyse approfondie des bienfaits de l'inoculation, réfute systématiquement les objections et expose ses propres succès en la matière. S'appuyant sur les médecins européens les plus reconnus de l'époque, il entend adapter à la Nouvelle-Espagne les expériences menées par Mead à Londres, par Chirac à Montpellier, par Molin à Paris et par Boerhaave à Amsterdam. L'inoculation n'étant toujours pratiquée en Espagne que semi-clandestinement par quelques praticiens éclairés liés à la médecine française ou britannique, Morel se réfère habilement aux milieux éclairés de la Société basque, dont le bulletin trimestriel de septembre 1776 rendait compte du succès de l'inocula-

(34) Liliana Schifter, Medicina, minería e Inquisición, op. cit., p. 30. Morel donne le détail de ses expériences en annexe de sa Dissertation.

(35) Archivo Histórico de la Ciudad de México [AHCM], Salubridad, vol. 3678, exp. 1 : « Aviso al Público », f. 1 (transcrit dans Liliana SCHIFTER, Medicina, minería e Inquisición, op. cit., p. 18).

(36) Esteban Morel, « Disertación sobre la utilidad de la inoculación » (AHCM, Salubridad, vol. 3678, exp. 2; reproduit en fac-similé dans Liliana SCHIFTER, Medicina, minería e Inquisición, op. cit., p. 66-193).

(37) Ibid. 
tion de 1200 brebis $^{38}$. Inversement, il n'hésite pas à critiquer ouvertement José Amar ${ }^{39}$, membre de la Haute Cour de médecine d'Espagne (Tribunal del Real Protomedicato) qui avait interdit en 1757 la publication de la traduction du mémoire de La Condamine ${ }^{40}$ et réprouvé en 1769 l'inoculation pour des raisons de santé publique. S'affranchissant des autorités de la métropole, Morel souligne que le climat favorable de la ville de Mexico était de nature à éviter la propagation des maladies et que, « comme dans les meilleures villes d'Europe », l'alimentation y est abondante toute l'année. Tenant compte de ces données locales, il propose la saison la plus favorable à l'inoculation et le régime le plus adéquat pour le patient, avant et après l'intervention. Il affirme de même que les personnes inoculées, une fois remises du traitement, n'attraperont plus la maladie et ne seront pas contagieuses.

Cependant, le succès médical de Morel et l'appui politique qu'il reçut se soldèrent à court terme par un échec du projet prophylactique. D'un côté, par méconnaissance et crainte du procédé, personne n'accourut se faire inoculer malgré l'annonce officielle et la clinique ferma ses portes peu de temps après. D'un autre côté, les membres du Protomedicato de Mexico se réservèrent la lecture de l'étude de Morel, dont les relations avec la mairie s'envenimèrent, aboutissant à un procès après le refus des autorités de payer le médecin et de publier son mémoire comme elles l'avaient promis. Enfin, les expériences de Morel arrivaient trop tard, au moment même où se terminait l'épidémie de 1779 , une des plus féroces de l'histoire de la Nouvelle Espagne ${ }^{41}$.

Le détail des activités professionnelles de Morel par la suite n'est pas connu, hormis quelques épisodes. Dès son installation à Mexico en 1778, il avait profité de la bonne réputation dont jouissait tout ce qui était français pour se constituer une clientèle privée. Il fut le médecin de famille et l'ami personnel de figures éminentes des élites administrative, économique et intellectuelle mexicaines, comme Juan Lucas de Lassaga, administrateur

(38) Ibid., § 107.

(39) Ibid., § 75, 92, 128, 129.

(40) « Memoria sobre la inoculación de las Viruelas de C.M. de La Condamine » (Archivo Histórico Nacional de España, Madrid, Consejos, liasse 50.653). Lu à l'Académie des sciences de Paris le 24 avril 1754, le mémoire de La Condamine fut imprimé en France, puis en Italie et en Angleterre. En Espagne, il fut traduit par Rafael Osorio en 1757 mais jamais publié. Juan Riera et Juan GRANDA-Juesas, La inoculación de la viruela, op. cit., p. 75.

(41) La mortalité doubla, voire quadrupla selon les lieux par rapport aux années antérieures. À Mexico, l'épidémie toucha 44286 personnes et causa 8820 décès (FernÁNDEZ DEL CASTILLo, Los viajes de Don Francisco de Xavier de Balmis, op. cit., p. 23). 
du Tribunal Royal des Mines (Real Tribunal de Minería), et le directeur de la Loterie et métallurgiste Francisco Xavier Sarría, déjà cité. Il eut aussi des liens étroits avec la communauté des apothicaires, collaborant « avec savoir-faire et soin, pour la composition de remèdes élégants, doux et efficaces » dans le laboratoire de certains d'entre eux, tels Antonio et Gregorio Mendez Prieto ou Antonio Lacodre et Antonio Arbide y Barroeta, à l'Hôpital général Saint-Andrét2. Mais n'hésitant pas à dénoncer le charlatanisme, il se faisait aussi des ennemis parmi confrères ${ }^{43}$.

En 1783, le médecin français demanda, sans succès, une place à l'hôpital des Indiens (Real Hospital de Naturales) de Mexico. Il attribua ce refus à un réflexe nationaliste des médecins locaux, refusant d'y voir employé un étranger non issu de l'Université royale et pontificale de Mexico $^{44}$. Mais une autre raison pesa probablement encore davantage sur la décision finale : sans qu'il en ait été informé, Morel faisait déjà, depuis l'année 1781, l'objet d'une procédure d'enquête au Tribunal du SaintOffice de l'Inquisition dont avait connaissance le Vice-Roi, personne de poids dans les décisions concernant l'hôpital royal.

\section{Les réticences de la société mexicaine : la constitution du dossier de l'Inquisition}

Morel avait acquis rapidement réputation, position sociale et argent, ce qui ne manqua pas d'exciter la jalousie d'une partie non négligeable de ses relations. Son esprit vif et volontiers moqueur, l'effronterie avec laquelle il s'exprimait sur la religion et son adhésion manifeste aux auteurs français matérialistes achevèrent de lui amener bien des ennemis. Lorsqu'advint le moment propice, ces derniers s'empressèrent de répondre à l'appel de l'Inquisition pour lui nuire.

Ouvert en 1781, le dossier inquisitorial d'Esteban Morel crût en volume au cours des quatorze années suivantes, jusqu'à sa mort et même au-delà. Constitué d'une série de dénonciations, d'extraits de sa correspondance, de dépositions relatives à ses livres et à des objets personnels, d'une part, et du témoignage de Morel lui-même tel que les greffiers de l'Inquisition l'ont retranscrit, d'autre part, ce dossier donne à voir la procédure inquisitoriale à l'œuvre et représente une source majeure sur le personnage, dont il éclaire les diverses facettes : son cercle social, sa vision

(42) Esteban Morel, « Solicitud... », f. 468.

(43) «Relación de la causa... », f. 269.

(44) AGN, Hospitales, année 1783, vol. 47, exp. 29 : Morel à la junte royale de l'hôpital, Mexico, 20 mars 1783, f. 474-478. 
de l'exercice de la médecine, sa vie personnelle, ainsi que ses idées scientifiques et sa position sociale.

L'origine de l'enquête de l'Inquisition sur Morel fut la plainte déposée en juillet 1781 par Ramon Barreyro, négociant de la capitale. Au cours d'une vive discussion, le médecin français aurait déclaré : «Pour moi, le mystère de la Divine Trinité est plus obscur que la nuit la plus sombre $»^{45}$. Des témoignages semblables expriment les doutes du médecin français, influencé par le vitalisme et le déisme. En 1785, Miguel Iturbide, officier de la Cour des Comptes, déclare l'avoir entendu dire : « Je laisse de côté le fait que l'Ame soit spirituelle mais elle doit être éternelle pour qu'elle puisse recevoir la récompense ou le châtiment en fonction de ses actes $[\ldots] »$ et ajouter 《 que Dieu pouvait engendrer une matière éternelle $»^{46}$. Morel doutait aussi de la nécessité de communier plus d'une fois l'an et de la véracité des textes sacrés. Affirmant que l'histoire des tremblements qui secouèrent la Terre à la mort de Jésus-Christ était apocryphe, il renvoyait ses interlocuteurs à Voltaire, dont la lecture était interdite par l'Église. Il manquait aux préceptes de l'Église Catholique, assurait que la simple fornication n'était pas contraire à la religion et qu'on ne lui ferait lire ni «l'Ancien testament ni le Coran parce que la foi du charbonnier me suffit $»^{47}$. Les dépositions accusatrices s'accumulèrent les mois suivants.

En avril, Joseph Ignacio Borunda, avocat de la Grande Chambre Royale (Real Audiencia Mayor) confirma les dépositions d'Iturbide. Déclarant n'avoir jamais vu Morel à l'église et le soupçonnant de vivre en concubinage, il précise que le Français lui a confié détenir « un extrait des œuvres de Volter (sic) », qu'il est « d'esprit osé et notoirement orgueilleux mais [que], peut-être par malice, il montre de l'indifférence pour donner son assentiment ou non aux opinions des autres sur les sujets qui ne sont pas dogmatiques $»^{48}$. En juillet, Juan de Santelices Pablo confirma que Morel lisait des livres interdits en le dénonçant pour détention d'un livre de « chimie » qu'il n'avait pas remis au Saint-Office. Entre autres accusations, le colonel Silvestre Lopez Portillo déclara en avril 1786, pour décharger sa conscience, que Morel avait refusé de préparer son ami et patient Juan Lucas de Lassaga à recevoir les sacrements chrétiens en alléguant que la peur qui en résulterait risquait de faire empirer l'état du malade. Ce

(45) Ibid., f. 229.
(46) Ibid.
(47) Ibid., f. 230.
(48) Ibid., f. 233. 
refus provoqua une grande agitation chez les parents et les amis de l'officier des mines, qui s'adressèrent aux autorités ecclésiastiques et réunirent un groupe de notables - dont faisaient partie José Ignacio Bartolache et Joaquin Velázquez de León entre autres - pour décider du sort de Larraga. Après bien du battage, Morel accepta de préparer ce dernier à recevoir à temps l'extrême onction ${ }^{49}$.

Ces plaintes contre Morel poussèrent l'Inquisition à convoquer des personnes citées dans ces premières dépositions, membres de l'armée et du clergé, fonctionnaires, gens du commun et domestiques. À leur tour, celles-ci mentionnèrent d'autres témoins possibles de Mexico, du Real de Catorce, de Guanajuato et même hors de la colonie. À ce stade, le magistrat de l'Inquisition fit remettre, le $1^{\mathrm{er}}$ septembre 1787, un extrait du dossier aux qualificateurs Gandarias et Palero de l'Ordre des Prédicateurs, lesquels en tirèrent la conclusion que :

« le Médecin français était un homme intrépide, orgueilleux, scandaleux, hérétique, Déiste, matérialiste, avec des apparences d'Athée, s'adaptant facilement à n'importe quelle religion, désobéissant à l'Église et aux lois des Princes, imprégné de beaucoup d'autres erreurs, comme celles qui consistent à avoir une mauvaise opinion de la Providence Divine, refuser la vénération des Images, condamner les actes extérieurs de la religion, mépriser les prières publiques, dénigrer et blâmer les pratiques de dévotion du Peuple Chrétien; erreurs qu'il avait bues sans doute à la source des Livres interdits, en particulier ceux de Wolter (sic) $[\ldots] »^{50}$.

Fort de ce premier rapport, le magistrat requit contre Morel la prison, la saisie des biens et «l'excommunication entre autres choses ». Mais, considérant le peu d'éléments à son encontre, il demanda un complément d'enquête et réclama des rapports à l'inquisiteur de Carthagène. L'épouse de Morel, Doña Agueda Maria Romero, était décédée depuis un ou deux ans mais la réponse du Saint Office du Venezuela en date du 31 août 1790 reprend son témoignage. Le principal motif de la plainte qu'elle avait déposée en mars 1771 était le rejet de la foi catholique par son mari qui, au bout de quinze jours de mariage, lui avait interdit de prier chez lui et refusait le baptême des enfants à venir. Selon elle, il tenait la foi maçonnique pour seule véritable et conservait jalousement un petit livre qui en renfermait les préceptes. Cette grave accusation passible de 
prison fut confirmée par des témoins des deux sexes ${ }^{51}$. Bien qu'aucune source n'atteste que Morel ait été initié, il n'est pas improbable qu'il ait été en contact avec des milieux maçonniques à Montpellier ${ }^{52}$ ou en Guadeloupe ${ }^{53}$ et sa signature tripunctée peut porter à le croire.

D'autres témoignages vinrent corroborer ces diverses accusations : Morel avait conseillé le mariage à une jeune femme qui voulait devenir nonne, déclaré que les guérisons attribuées à un miracle de Dieu par l'intercession des saints ne devaient l'être qu'à l'habileté des médecins, etc. ${ }^{54}$. Mais l'avalanche des dépositions orienta bientôt également vers le domaine politique les soupçons de l'Inquisition, de plus en plus méfiante envers ce qui touchait à la Révolution française.

\section{Morel et la Révolution française}

Convoqué par le Tribunal le 12 janvier 1790, le Dr. Joseph Garcia Brabo, un habitué du cercle du médecin français, déclara que celui-ci détenait des documents concernant les nouvelles de France et faisait circuler un texte de lui intitulé «Clé de ces révolutions » ${ }^{55}$. Brabo n'avait pas vu cet écrit, mais il remit au magistrat un autre document de Morel qu'il avait copié :

« un papier manuscrit en forme de Journal qui contient des informations sur les révolutions de Paris, en partie en castillan et en partie en Français, dans lequel il y avait beaucoup de venin et de propositions très discordantes et séditieuses, et qui renferment les principes et maximes funestes de la Philosophie Antichrétienne [...] $\gg^{56}$.

Il s'agissait d'un recueil d'extraits de lettres de Paris, du 30 novembre 1788 au 26 avril 1789, et du Journal de Paris des 15 et 30 août $1789^{57}$.

(51) «Sobre los asuntos... », f. 267.

(52) Arrivé pour ses études à Montpellier le 4 mars 1757, Pierre-Jacques Willermoz appartient déjà à une loge locale un mois plus tard (Lehman, Venel..., p. 97).

(53) Voir Alain Le BiHAN, « La Franc-Maçonnerie dans les colonies françaises du XVIII siècle », $A H R F, 46$ (1974), p. 39-62. Maurel et sa famille sont néanmoins absents du dictionnaire d'Élisabeth Escalle et Mariel Gouyon-Guillaume, Francs-maçons des loges françaises aux Amériques. Contribution à l'étude de la société créole, Paris, E. Escalle, 1993.

(54) «Relación de la causa... », f. 269 (témoignage de la comtesse de San Pedro del Alamo et de Dona Maria Francisca Gorraez).

(55) Ibid., f. 251. Le Diccionario Porrúa attribue abusivement sa condamnation à la diffusion de ce texte.

(56) Ibid., f. 250v.

(57) Ibid., f. 251-253v. 
Ces extraits ne comportaient aucun mot hostile envers la monarchie espagnole, l'Église catholique ou le Saint-Office mais les lettres reflétaient les espoirs mis en Necker et dans les États généraux et évoquaient avec émotion la fin de la tyrannie, du despotisme et de l'aristocratie et des « barbares privilèges ». Le Tribunal ordonna aussitôt une perquisition dans les papiers de Morel.

Dans la nuit du 13 janvier 1790, dix-neuf lettres et six documents écrits en français entre 1787 et 1789 furent saisis chez lui, traduits et analysés par le chanoine pénitencier Josef Patricio Uribe, expert qualificateur du Saint-Office ${ }^{58}$. Les lettres - celles-là mêmes dont Morel avait copié des extraits dans le document livré par Brabo - provenaient principalement de son frère « Honorato » et de son neveu Darboussier, alors à Paris, mais demeurant ordinairement en Guadeloupe ${ }^{59}$. Ce dernier était 1'un des trois fils de Jean Darboussier (1737-1803), l'un des principaux négociants de Pointe-à-Pitre. Également natif d'Aubagne et installé en Guadeloupe vers 1770 , le père était notamment en affaires avec Bordeaux mais la Guerre d'indépendance des États-Unis fit fleurir ses affaires avec la Nouvelle-Angleterre et New York et la maison de commerce se lança même dans la course ${ }^{60}$. Comme la plupart des négociants de Pointeà-Pitre, les Darboussier allaient devenir des patriotes proches des autorités de Port-de-la-Liberté en $1794^{61}$. Mais pour 1'heure, aucune de ces lettres n'était véritablement compromettante pour Morel et l'expert du Saint-Office souligne même que l'accusé termine par une phrase « très chrétienne » une lettre à sa sœur Clairon, à propos des espoirs matrimoniaux de celle-ci ${ }^{62}$.

La plupart des lettres portent en effet sur des sujets domestiques et privés. Son frère Honoré parle surtout de ses affaires commerciales et de son désir de venir s'installer avec lui et s'enrichir dans les mines ${ }^{63}$.

(58) Ibid., f. 253v-262v.

(59) Probablement lié à la maison de commerce Durand, Maurel \& $\mathrm{C}^{\mathrm{ie}}$, le conseiller Honoré Maurel avait ouvert, à Pointe-à-Pitre en 1778, une rue portant son nom, à côté de celle du gouvernement. Anne Pérotin-Dumon, La ville aux îles, la ville dans l'île: Basse-Terre et Pointe-à-Pitre, Guadeloupe, 1650-1820, Karthala, 2000, p. 402.

(60) Ibid., p. 198.

(61) Anne Pérotin-Dumon, La ville aux îles...,p. 198. L'aîné des neveux de Morel fut envoyé à Charleston pour organiser le ravitaillement de la Guadeloupe encerclée par les Anglais; sa nièce épousa un proche du commissaire de la Convention Victor Hugues, le général Boudet, commandant de la place puis des troupes de l'île; les autres frères vécurent dans l'entourage des autorités ou occupèrent des fonctions publiques.

(62) « Relación de la causa... », nº 2 et 9, f. 257 et 259.

(63) Ibid., n 3, f. 257-258. 
"Quelques points physiques, et de minéralogie» - en particulier sur le platine, monopole espagnol - sont abordés par son neveu ${ }^{64}$ et les échanges avec le Baron Ignaz von Born, savant métallurgiste et francmaçon autrichien, concernent son livre sur la production des métaux, que le roi d'Espagne avait envoyé à Fausto de Elhúyar pour qu'il utilisât sa méthode ${ }^{65}$.

Tout au plus, une lettre faisant référence au Dictionnaire philosophique de Voltaire compare les lois monarchiques à l'Inquisition espagnole et témoigne de l'espoir mis dans les États généraux : «Alors il n'y aura plus de tyrans de la raison, il y aura la liberté totale de parler et d'écrire ${ }^{66}$. D'autres détaillent les événements du printemps et de l'été 1789, témoignant des espoirs mis dans le « second Sully » ou « second Protestant sauveur de la France, M. Necker » et dans la liberté de la presse, ou annonçant la prise de Bastille, la mort de Launay, de Foulon et de Bertier et l'émigration des princes ${ }^{67}$. La correspondance mentionne aussi Vives, un «vieil ami au Mexique » séjournant à Paris, sans nul doute un créole chaud partisan de la Révolution ${ }^{68}$.

Enfin, une brochure française annotée par le médecin fut également saisie lors de la perquisition. Sous le titre « Sommaire des articles convenus le 4 août », elle porte sur les sujets très sensibles de l'abolition des privilèges, de l'égalité devant l'impôt, du rachat des droits féodaux, de l'abolition des justices seigneuriales et de la vénalité des offices, de l'admission de tous les citoyens aux charges civiles et militaires, etc ${ }^{69}$. Aux vingt-deux articles, dont Morel souligne qu'ils sont appelés à être modifiés par l'Assemblée, il ajouta des notes manuscrites extraites de lettres ou de journaux, fournissant au lecteur des informations sur le contexte : «Mr. Bailli de l'Académie française, et de celle des sciences, qui fut président de l'Assemblée pendant les 15 premiers jours, a été nommé maire de Paris par nomination de la Ville, confirmée par le Roi.

(64) Ibid., $\mathrm{n}^{\circ} 3$ et 11, f. 257 et 259-260.

(65) Ibid., $\mathrm{n}^{\circ} 2$ et 10, f. 257 et 259. José Antonio Alzate rapporte dans son périodique que ce fut Morel qui l'informa de la méthode de Born et de l'œuvre de cet auteur. Voir Jose Antonio Alzate, « Para beneficiar los metales », Gacetas de Literatura, op. cit., t. 4, p. 203.

(66) Ibid., $\mathrm{n}^{\circ} 4$, f. $257 \mathrm{v}-258$.

(67) Ibid., lettres du neveu, $\mathrm{n}^{\circ} 11,20$ et 21 , f. 259-262. Le frère donne aussi quelques nouvelles politiques ( $\mathrm{n}^{\circ} 12$ et $\left.16,260-260 \mathrm{v}\right)$.

(68) Ibid., $\mathrm{n}^{\circ} 5$ et 7, f. 258. Voir Luisa Zahíno PeÑAfort, « El criollo mexicano Francisco Vives y su correspondencia desde la Francia revolucionaria : de canónigo catedralicio a miembro de una sociedad jacobina », Estudios de Historia Novohispana, n ${ }^{\circ}$ 15, 1995, p. 113-127.

(69) Il doit s'agir du Sommaire des articles convenus le 4 août 1789 pour former la Constitution, Paris, Debray, s.d. (BnF Le $\left.{ }^{29} 104\right)$. 
Il a succédé dans cette charge à M. Fleselles (sic), prévôt des marchands, égorgé pour cause de trahison. M. Bailli a présenté au Roi les clés de la ville $[\ldots] »^{70}$.

Ces documents ne constituaient toujours pas vraiment un motif suffisant pour emprisonner Morel, mais le Tribunal les conserva et les utilisa quelques années plus tard lors de son procès. Entre-temps, les circonstances avaient changé, les Espagnols craignant la contagion révolutionnaire, surtout après la chute de la monarchie et la mort de Louis XVI.

\section{L'arrestation et le procès de Morel}

La tension croissante entre les nations espagnole et française se faisait également sentir dans les colonies américaines. L'effervescence politique des premières années se transforma en manifestations de méfiance. Des rumeurs de conspirations fomentées par les habitants d'origine française, alimentées par le gouvernement et le clergé, se répandirent par les villes, entraînant délations et persécutions et une paranoïa presque délirante ${ }^{71}$, point d'orgue de la sévérité et l'énergie avec lesquelles, dans le dernier quart du siècle, l'Inquisition chercha à protéger la monarchie espagnole de la contagion des Lumières qui menaçaient la souveraineté du Roi et la toute puissance de l'Église. Des chaires jaillirent alors des appels enflammés à la fidélité envers la Couronne espagnole. Les sermons vilipendaient les philosophes et révolutionnaires français :

« [...] un groupe de philosophes séducteurs et malveillants, cruels et sanguinaires, qui prétend détruire l'ordre que Dieu a établi depuis le début pour gouverner l'univers et qui a eu la sacrilège audace de renverser le trône et de sacrifier à sa fureur les vies précieuses et sacrées de leurs légitimes souverains $»^{72}$.

Les nouvelles de la mort de Louis XVI (21 janvier 1793) et de la déclaration de guerre contre l'Espagne (7 mars), parvenues en NouvelleEspagne en juin, mirent la colonie en état d'alerte. L'année suivante, la

(70) « Relación de la causa... », f. 254v-256v.

(71) Gabriel Torres Puga, « Centinela mexicano... », op. cit., p. 1.

(72) Juan de Sarría y Alderete, Sermón moral sobre el Evangelio de la Dominica Infraoctava de Epifanía predicado en presencia del Excmo. Señor virrey de esta Nueva España y de la Real Audiencia de México en el Real convento de religiosas de Jesús María..., México, Joseph de Zúñiga y Ontiveros, 1795. 
Couronne se prit à croire que la guerre pouvait faire naître des sentiments francophiles et des opposants au gouvernement dans les colonies. Le 29 juillet 1794, un libelle alarmant dans la Gazeta de México amalgamait la domesticité française aux jacobins de Paris : "Ces monstres sont déjà parmi nous et se sont infiltrés dans nos familles $\gg{ }^{73}$. La peur des autorités culmina le 24 août, avec l'apparition dans la capitale - cinq jours après Bogota - de pasquins faisant l'éloge de la liberté de la République française. Le Vice-Roi Branciforte, beau-frère de Manuel Godoy, venait de prendre son poste à Mexico le 12 juillet. Prenant le contrepied de la politique du comte de Revillagigedo, son prédécesseur, la sienne conduisit à la persécution des Français et des révolutionnaires supposés ${ }^{74}$. Il donna ordre au Tribunal criminel (Real Sala del Crimen) et à celui de l'Inquisition de trouver les auteurs « séditieux » et chargea le maire de Mexico, Joaquín Romero de Caamaño, de chercher s'il était vrai que de nombreux Français se réunissaient en clubs dans la ville pour discuter de la politique de leur patrie. Des poursuites judiciaires contre dix-sept Français et autres individus de la ville furent lancées en septembre et octobre. Au nombre des personnes arrêtées furent Morel et un autre propriétaire de mine d'argent, Juan Fournié [Jean Fournier] et des tenanciers de cafés et billards où les Français se réunissaient. Loin d'être des idéologues subversifs, les autres étaient des artisans ou des domestiques de grandes familles de la noblesse et du négoce, comme le cuisinier du Vice-Roi, Juan Lausel, ou le perruquier Vicente Lulié [L'huillier], valet de chambre de l'assesseur du Vice-Roi ${ }^{75}$. Leur faute était surtout d'avoir commenté en privé les rares informations générales que le courrier leur apportait de France, via Philadelphie, car les accusations portées contre eux étaient fondées moins sur des preuves dignes de foi que sur la paranoïa, la xénophobie et l'ignorance ambiantes $^{76}$.

Le 5 septembre, Morel fut incarcéré dans les prisons secrètes de l'Inquisition. Dès le lendemain, une audience préliminaire porta sur «l'actuelle révolution de la France ». Lors de cette première comparution, il essaya de biaiser en disant qu'il ne trouvait rien à répondre aux

(73) Cité par Antonio IBARRA, «Conspiración, desobediencia social y marginalidad... », p. 27 (n. 4$)$.

(74) Luís Navarro García, "México en la política de Godoy », Revista de Estudios Extremeños, vol. 57, n 3, 2001, p. 1155-1168.

(75) Archivo Histórico Nacional, Madrid, Estado, liasse 4178, 2ª . Voir Gabriel TorRes PugA, «Centinela mexicano... », p. 65.

(76) Gabriel Torres PugA, « Centinela mexicano... », p. 65. 
questions et qu'il le ferait plus tard si la mémoire lui revenait. De nouvelles déclarations confirmèrent bientôt les accusations antérieures et, sur demande expresse présentée au Vice-Roi par la Sainte Inquisition en octobre, la correspondance des suspects fut interceptée. Dans cette ambiance de psychose collective, Branciforte donna l'ordre aux intendants des provinces, le 10 décembre 1794, d'arrêter tous les Français de Nouvelle-Espagne, de saisir leurs biens et de les incarcérer à la prison de la Cour de Mexico. À la fois juge et partie, Borunda - l'un des accusateurs de Morel en 1785 - servit d'interprète dans les premières confrontations avec les accusés mais il apparut ensuite qu'il maîtrisait mal le français et que les conclusions qu'il tirait de l'analyse des documents sous séquestre, toujours au préjudice des accusés, étaient totalement infondées ${ }^{77}$. Les accusés restèrent néanmoins en prison durant toute la guerre, perdus dans la jungle des dossiers et des procès entachés d'irrégularités par le manque de compétence et l'incessant renouvellement du Tribunal et des autorités.

Le procès de Morel s'étala du 3 octobre 1794 au 11 février 1795. La retranscription des quatre audiences montre que le ton et la gravité des accusations montèrent peu à peu pour enserrer l'accusé dans un étau de plus en plus violent. À la première, les 3 et 4 octobre $^{78}$, le prisonnier déclina son identité, déclara avoir vécu « librement avec Doña Maria de Ribera » de 1779 à 1786 , et prétendit ne s'être jamais écarté des préceptes catholiques, ni dans son comportement ni à propos des événements de France.

La seconde audience commença les 23 et 25 octobre et prit fin le 10 novembre ${ }^{79}$. Si Morel reconnut avoir manqué une dizaine de fois à l'accomplissement des devoirs de l'Église, il déclara l'avoir fait non par mépris des sacrements, mais, au contraire, par respect pour eux. Au sujet de ses papiers relatifs à la Révolution française, il admit avoir extrait des morceaux de quelques « courts chapitres concernant ladite révolution » pour les communiquer à ses compagnons « $\mathrm{D}[\mathrm{o}]^{\mathrm{n}}$ Miguel Constanzo, $\mathrm{D}^{\mathrm{n}}$ Fran[cis]co Sarria, $\mathrm{D}^{\mathrm{n}}$ Vicente Zervantes, $\mathrm{D}^{\mathrm{n}}$ Mig[ue]l Pacheco, $\mathrm{D}^{\mathrm{n}}$ Fausto de Éluiar et au Dr Brabo, et à d'autres; lesquels en amenaient habituellement d'autres concernant la situation en France, avant et après leur interdiction par le Saint-Office $\gg{ }^{80}$. Il précisa néanmoins que ces

(77) Ibid.

(78) «Relación de la causa... », f. 269v-270v.

(79) Ibid., f. 270v-273v.

(80) Ibid., f. 271. 
sujets étaient abordés dans les réunions par simple passe-temps, que «ni les assistants ni [lui] ne montrèrent un esprit de sédition », et qu'aucune plainte, réclamation ou réprobation contre la monarchie espagnole n'y fut jamais exprimée. Il insista sur le fait que tous ceux avec lesquels il avait discuté familièrement de ces sujets réprouvaient les actions violentes que l'Assemblée et le peuple de Paris avaient commises contre le genre humain, la religion et l'autorité.

Au nombre des personnes impliquées dans cette circulation d'informations clandestines figurait Joseph Manuel de los Reyes, un propriétaire foncier de la juridiction de San Juan de los Llanos. Les soupçons du Tribunal furent éveillés par les échanges épistolaires et les relations familières qu'il entretenait avec Morel. L'Inquisition ordonna une enquête sur lui. Interrogé au sujet de sept lettres, du 20 novembre 1791 au 2 mai 1793, probablement réquisitionnées chez Morel, Reyes affirma qu'elles ne contenaient rien contre la religion ou le gouvernement espagnol, et qu'on n'y pariait aucunement sur la mort du roi de France. Il déclara aussi ignorer qui était le correspondant de Morel en Espagne et en NouvelleEspagne. Il ressort de ses déclarations que Morel lui avait transmis plusieurs papiers comportant des nouvelles de France tirées de gazettes ou obtenues par des amis. Le témoin déclara avoir commencé à les conserver, avant de les brûler lorsqu'il apprit l'interdiction de faire circuler les nouvelles en provenance de France. À défaut de fournir le nom d'autres correspondants, ces lettres témoignent de l'existence d'un réseau d'informations sur la Révolution française dans lequel Morel était actif.

Le 18 novembre eut lieu la troisième audience ${ }^{81}$. Le prisonnier remit un feuillet dont il était l'auteur, dans lequel il répondait aux accusations portées contre lui pour avoir fait circuler la Défense de Louis XVI devant la Convention, par l'avocat de Sèze, avec ses propres réfutations manuscrites. L'un des témoins dit avoir vu le botaniste Vicente Cervantes lire de document.

\section{Buffon contre la Genèse}

La quatrième audience fut sans doute la plus difficile ${ }^{82}$. Elle débuta le 6 février 1795, par l'annonce des cent-trente chefs d'accusation liés aux diverses dénonciations antérieures, qui furent ensuite réexaminés un à un. Tantôt Morel répondait par des objections argumentées, tantôt 
il se contentait de déclarer que les accusations étaient fausses. Les chefs d'accusation 19 à 21 portaient sur ses croyances non conformes aux Saintes Écritures.

Déjà accusé d'avoir lu des livres interdits et copié des passages de lettres citant Voltaire, Rousseau, d'Alembert ou d'autres auteurs modernes qui, sans concerner la religion, décelaient des vues matérialistes et hérétiques ${ }^{83}$, Morel avait reconnu, lors de la deuxième audience, avoir lu en France et aux Antilles «Rousseau, Voltaire, Montaigne, Montesquieu, Raynal et probablement quelques autres œuvres qui seraient interdites dans les zones où il y a l'Inquisition $»^{84}$. Mais il avait nié l'avoir fait durant ses seize années de résidence à Mexico. Cette fois, l'audience porta plus particulièrement sur les opinions de Buffon sur la formation de la Terre.

De nombreux témoignages attestent en effet que Morel connaissait l'œuvre de Buffon. À Nicolas Aviles, qui l'interrogeait sur la cause des «tremblements souterrains", il avait répondu que lui «croyait que c'était du feu » car au début du monde un morceau de soleil se détacha et forma la terre, comme le soutenait «Buffon, célèbre auteur français » qui affirmait aussi que "l'ensemble de la création n'avait pas duré des jours comme l'affirmaient les Écritures mais des siècles et des siècles $»^{85}$. Dans la chronologie de la Terre du premier tome de son Histoire naturelle (1749), Buffon ne se réfère pas à la Genèse mais considère que la vie sur la terre est le résultat d'une série d'événements cosmiques. Trente ans plus tard, dans Les Époques de la Nature, il divise l'histoire de la terre en sept époques : dans la première, elle n'était qu'une masse fondue arrachée du Soleil par le choc d'une comète il y a 75000 ans.

L'ouvrage était paru à Paris en 1778. En s'y référant explicitement dans sa défense, Morel avoue donc implicitement l'avoir lu en NouvelleEspagne. Bien que la lecture de Buffon ne fût pas formellement interdite par l'Église catholique, elle était considérée par les inquisiteurs comme opposée aux textes sacrés et à la religion catholique. Utilisant des arguments typiques des matérialistes, qui soutenaient que l'homme est un être purement matériel, que la faculté de penser provient de la matière et que l'âme est une invention humaine inutile, Morel déclara que Dieu pouvait créer une substance matérielle et éternelle. À ces affirmations, il jugea bon

(83) Ibid., f. 262-262v.

(84) Ibid., f. 270v-273v.

(85) Ibid., f. 246v. 
d'ajouter qu'il n'y avait pas d'« inconvénient à donner à ce système et à son auteur le degré de probabilité qu'ont les recherches philosophiques ». Pour sa défense, il argua aussi que Buffon avait fourni à ce sujet les explications satisfaisantes et surtout sa soumission à la Sorbonne et que, puisque celle-ci et le Saint-Office ne s'opposaient pas à la libre circulation de son œuvre, il en était de même pour les Époques de la Nature ${ }^{86}$.

Dans l'échauffement de ses réponses, Morel avoua son admiration pour Buffon, « son talent, son érudition, son style et surtout de sa soumission la plus respectueuse aux livres sacrés $\rangle^{87}$. Il osa même ajouter que le mot « jour » dans la Genèse signifiait un intervalle de temps indéterminé dans la création, et pouvait représenter les années ou les siècles nécessaires pour que la terre, sortant des mains de Dieu, eût la perfection nécessaire. Les inquisiteurs objectèrent que, même si les recherches purement philosophiques étaient permises, « les croyances ne devaient jamais se laisser gouverner par elles » et qu'accepter le fait qu'il ne s'agissait pas de jours mais d'années et de siècles de création était une hérésie. Arrivée à ce point, l'audience fut levée.

Dans son ultime défense devant le Tribunal, Morel arriva disposé à ne pas renier le système de Buffon. Il déclara que, avant même sa naissance, l'œuvre de Buffon était «tout à fait autorisée » et diffusée dans toute l'aire chrétienne, et qu'elle se trouvait dans les librairies sans opposition des autorités. Pour compléter sa défense, il argua que l'Église avait accepté les réfutations de Galilée, considérant que les paroles des Écritures qu'on lui opposait pouvaient bien « ne plus être prises à la lettre ». Selon Morel « le destin des livres sacrés [n'était pas] d'enseigner au peuple la physique, mais seulement de fixer les matières de croyances $»^{88}$. En conséquence, ni son adhésion au système de Buffon, comme une conjecture physique probable, ni le fait que les intervalles de création ne fussent pas des jours mais des années ou des siècles ne lui avait donc semblé contrevenir à la croyance catholique.

La conséquence immédiate et prévisible de cette argumentation fut un nouveau chef d'inculpation pour « croyance erronée et persistance dans celle-ci ${ }^{89}$. Tout en connaissant le danger auquel il s'exposait, Morel ne renia pas ses croyances matérialistes ni son adhésion au système

(86) Ibid., f. 277.

(87) Ibid., f. $277 \mathrm{v}-278$.

(88) Ibid., f. 280.

(89) Ibid., f. $281 \mathrm{v}$. 
buffonien ${ }^{90}$. Pour sa défense, il rappela que, dans son explication de la Genèse, Buffon attribuait à la pauvreté de la langue hébraïque le fait que le mot «jour» puisse parfois être interprété dans un sens indéterminé désignant un intervalle de temps de manière générique. Le fait que luimême ait déclaré que le mot avait un sens indéterminé n'excluait donc pas le sens de jour naturel et il n'était pas dans ses intentions d'offenser la croyance catholique. Puis il demanda une suspension de séance, car il avait « le cerveau fatigué et tourmenté ».

Six mois s'étaient écoulés depuis son emprisonnement. Sa santé et son courage faiblissaient, par suite des conditions de détention et des nouvelles alarmantes qui circulaient sur le sort de ses compatriotes. De fait, Fournié, accusé d'utiliser sa mine d'argent pour financer le soulèvement, avait été soumis à deux atroces séances de torture par le Saint-Office : le 12 février 1795 le médecin et le chirurgien de la prison royale constatèrent une grave blessure, dont il mourut quelques semaines plus tard ${ }^{91}$. À l'issue de cinq audiences épuisantes, seulement $15 \%$ des accusations avaient été lues. Le procès de Morel s'arrêta pourtant là. Le 15 février 1795 les gardes de la prison, ayant trouvé sa cellule verrouillée de l'intérieur et la fenêtre fermée, forcèrent l'entrée : « ils trouvèrent tout recouvert de sang et ledit prisonnier assis au pied de son lit, baignant dans son propre sang $\rangle^{92}$. Morel semble s'être perforé la carotide à l'aide d'une mouchette. Il était encore en vie, mais ses geôliers, plus préoccupés de son âme que de son corps, lui refusèrent une assistance médicale avant qu'il se fût confessé, ce qui fut fait au bout d'une heure et des efforts conjugués de trois prêtres. Quand ils tentèrent enfin de l'aider, c'était déjà trop tard. Il expira à dix heures et demie du matin et fut enterré le jour même en l'église du chapitre de Santo Domingo.

La mort de l'accusé ne suffit pas à satisfaire le tribunal. L'Inquisition décida de poursuivre pour hérésie et... suicide le procès contre « la mémoire et la réputation dudit Morel conformément au Droit et à la manière du Saint-Office et que soient assurés ses Biens ». Enfin, le 19 juin 1795, les inquisiteurs déclarèrent Esteban Morel « Hérétique

(90) Le prêtre Juan Antonio de Olavarrieta, inculpé pour matérialisme en 1802-1804, se référait également à Buffon. Elías TRABulse, «Aspectos de la diffusion del materialismo científico de la Ilustración francesa en México a principios del siglo XIX », dans Solange AlBerro et al., La Revolución Francesa en México, p. 81-96.

(91) Voir Gabriel Torres PugA, « Centinela Mexicano... », p. 88-89.

(92) «Relacion de la causa... », f. 282-283. 
patenté et suicidé », ordonnèrent que ses biens acquis depuis le mois de juillet 1778 fussent confisqués, qu' ' on fasse une statue à l'effigie du prisonnier, qu'elle soit présentée lors du premier Jugement public, revêtue des insignes de Pénitent et Hérétique patenté $\rangle^{93}$. Le 9 août, cet acharnement prit fin avec l'autodafé de Juan Lausel en l'église Santo Domingo, qui impliqua également Juan Murgier, un autre inculpé suicidé ${ }^{4}$.

$*$

Le changement du paysage politique après le traité de Bâle entre la France et l'Espagne ( 22 juillet 1795 ) et l'excellent travail des avocats de la défense retardèrent les jugements des prisonniers et permirent la révision des procès, réduisant la théorie du complot révolutionnaire à une chimère. Les prisonniers survivants furent néanmoins remis à l'Espagne.

La mort de Morel et le dénouement posthume de son procès ne font pas toute la lumière sur une série d'interrogations depuis l'origine jusqu'à son apparent suicide. Pourquoi ses compagnons de réunion ne furent-ils pas convoqués, hormis le Dr. Brabo, témoin à charge particulièrement zélé ? Le fait que Morel ait possédé une mine d'argent eut-il une influence sur l'acharnement particulier dont il fut victime, à l'instar de Fournier, l'autre Français propriétaire minier? Le litige concernant sa propriété de la mine du Morán eut-il un lien quelconque avec cette affaire? Quelques mois après la condamnation posthume, en tout cas, Joseph Belio, son rival débouté en 1793, en était devenu propriétaire.

Sans être un conspirateur, Morel fut néanmoins, comme son confrère Louis Rieux à Bogota, un élément important du réseau de diffusion des nouvelles venues de France. Son appartenance à un cercle savant éclairé, lié aux élites politiques et économiques de la vice-royauté, ne lui permit pas d'échapper aux persécutions de Branciforte mais elle lui offrait une sphère d'influence qui pouvait, par l'intermédiaire de ses compagnons, s'étendre à d'autres couches de la société, en particulier auprès des nouvelles générations qui accouraient dans les nouvelles institutions académiques où plusieurs enseignaient. Ses propres travaux pionniers en matière d'inoculation prennent toute leur importance tant au

(93) Ibid., f. 286.

(94) Gabriel Torres Puga, Opinión pública, op.cit., p. 471. 
regard du retard de la péninsule qu'à celui de la nouvelle épidémie générale de 1797. Au moment même où Morel croupissait dans les geôles de l'Inquisition et polémiquait avec ses juges, le contexte national et les mentalités avaient commencé à changer : 70000 personnes furent traitées en Nouvelle-Espagne entre 1794 et 1798 - soit autant qu'un pays aussi peuplé et éclairé que la France durant tout le siècle - ce qui limita les effets de la nouvelle épidémie générale de $1797^{95}$. Le Mexique avait plus que rattrapé son retard.

Liliana Schifter et Patricia Aceves Université Autonome Métropolitaine-Xochimilco Mexique (UAM-X) 1schif@correo.xoc.uam.mx paceves@correo.xoc.uam.mx

Patrice BRET Centre Alexandre Koyré Centre de recherche en histoire des sciences et des techniques (UMR 8560 du CNRS) patrice.bret@yahoo.fr

(95) Robert McCAA, «Inoculation : An easy means of protecting people or propagating smallpox? Spain, New Spain and Chiapas, 1779-1800», Boletín de Historia y Filosofia de la Medicina, vol. 2, sept. 1998. Les autorités attribuèrent à cette inoculation massive la réduction considérable du nombre de morts (AHCM, Junta Superior de Sanidad de México, 1824 : 14). 